\title{
Features associated with underlying HIV infection in severe acute childhood malnutrition: a cross sectional study
}

\author{
James Bunn ${ }^{1,2}$ Miriam Thindwa ${ }^{3}$ Marko \\ $\operatorname{Kerac}^{1,4,5}$. \\ 1 College of Medicine, Blantyre, Malawi \\ 2 Liverpool School of Tropical Medicine, UK \\ 3 District Health Office, Blantyre, Malawi \\ 4 UCL Centre for International Health and Development, London, UK \\ 5 Valid International, Oxford, UK
}

\section{Abstract}

\section{Introduction}

Up to half of all children presenting to Nutrition Rehabilitation Units (NRUs) in Malawi with severe acute malnutrition (SAM) are infected with HIV. There are many similarities in the clinical presentation of SAM and HIV. It is important to identify HIV infected children, in order to improve case management. This study aims to identify features suggestive of HIV in children with SAM.

\section{Methods}

All 1024 children admitted to the Blantyre NRU between July 2006 and March 2007 had demographic, anthropometric and clinical characteristics documented on admission. HIV status was known for 904 children, with 445 (43\%) seropositive and $459(45 \%)$ seronegative. Features associated with HIV were determined.

\section{Results}

Associations were found for the following signs: chronic ear discharge (OR 14.6, 95\% CI 5.8-36.7), lymphadenopathy (6.4, 3.5-11.7), clubbing (4.9, 2.6-9.4), marasmus (4.9, 3.56.8), hepato-splenomegally (3.2, 1.8-5.6), and oral candida $(2.4,1.8-3.27)$. Any one of these signs was present in $74 \%$ of the HIV seropositive, and $38 \%$ of HIV uninfected children. A history of recurrent respiratory infection (OR 9.6, 4.8-18.6), persistent fever, recent outpatient attendance, or hospital admission were also associated with HIV. Persistent diarrhoea was no more frequent in HIV (OR 1.1). Orphaning (OR 2.1, 1.4-3.3) or a household contact with TB (OR 1.7, 1.1-2.6), were more common in HIV. Each of these features were present in $>10 \%$ of seropositive children. HIV infected children were more stunted, wasted, and anaemic than uninfected children.

\section{Conclusions}

Features commonly associated with HIV were often present in uninfected children with SAM, and HIV could neither be diagnosed, nor excluded using these. We recommend HIV testing be offered to all children with SAM where HIV is prevalent.

\section{Introduction}

$90 \%$ of childhood HIV occurs in Africa, and is associated with $7 \%$ of all deaths in children aged under 5 (1). Severe acute malnutrition (SAM) is a common presentation of HIV(2) in Malawi, with up to half of children admitted to nutrition rehabilitation units (NRUs) seroposive for HIV (3).
In a metanalysis of African studies on SAM, overall mortality in HIV infected children was $30.4 \%$, compared with $8.4 \%$ in HIV negatives (4). HIV infected children may have different care needs during admission(5), and community interventions such as cotrimoxazole (6) and ARVs (7) can reduce their long term mortality. Parental diagnosis and treatment may also be relevant, reducing orphaning and improving child survival (8). To access these services HIV infection needs first to be identified.

There are many similarities in the clinical presentations of SAM and HIV. This is partly due to a cell mediated immunodeficiency in SAM, $(9,10)$, but also due to malnutrition arising from poor appetite and a catabolic state in advanced HIV (11). A cycle of infection, malnutrition and immunodeficiency has been described (12). Once within this cycle, many of the clinical manifestations are similar. Previous studies have attempted to identify which clinical features are suggestive of underlying HIV infection in malnutrition (13), with adenopathy, oral candida and marasmus being most sensitive. The child's parental health and past medical history have not been evaluated previously.

Some of the clinical features recognized in SAM, such as oral candida, are staging criteria for $\mathrm{HIV}(14)$ or markers of HIV in presumptive diagnosis of infants(14), including malnutrition itself. Nutritional features which may help identify underling HIV in a child with SAM include wasting (marasmus), reported to be 4-6 times more common in HIV infection $(3,13,15-20)$, and a weight $<60 \%$ of the expected median for age (18).

Oral candidiasis is more common in $\operatorname{HIV}(2,13,21-24)$, though not in all studies in malnutrition (18). Chronic suppurative otitis media (CSOM) has been reported as more common in HIV infection, both on pediatric wards (2), and in the NRU (24).

Diarrhea is common in children presenting with SAM, with enteropathy and persistent diarrhea (PD) common in HIV $(21,25)$, though intestinal helminths appear equally prevalent $(26,27)$. Clubbing, indicative of chronic lung disease (CLD) is seen in HIV (2), though not specifically reported in SAM. Lymphadenopathy $(13,24)$ has been associated with HIV in children presenting with SAM. Anemia occurs more commonly, with a mean hemoglobin of $8.3 \mathrm{~g} / \mathrm{dl}$ in HIV, compared to $8.8 \mathrm{~g} / \mathrm{dl}$ in uninfected children with SAM (28).

The aim of this study is to describe the clinical, and demographic associations of SAM with, and without, underlying HIV. These features could play a role in identifying children with HIV, so the diagnosis of HIV can be confirmed, and children directed to specific HIV pathways as early as possible.

\section{Methods}

All children admitted to the Queen Elizabeth Central Hospital NRU, Blantyre, Malawi, between July 2006 and March 2007 were included in the study. It was nested within a randomized trial on improved foods for $\operatorname{SAM}(29)$. As part of the trial, all 
children were assessed in detail at admission.

Admission criteria were: Weight for Height (WH) $<70 \%$ of median NCHS reference, a Mid Upper Arm Circumference (MUAC) $<110 \mathrm{~mm}$, or the presence of bilateral non-pitting oedema (kwashiorkor) (30). Children were weighed on Tanita 1582 digital scales, and length and MUAC were measured to the nearest $1 \mathrm{~mm}$ using locally made height boards and MUAC tapes (UNICEF).

HIV counseling and testing (HCT) was offered for all children, usually after initial stabilization. This involved two ELISA rapid tests (Determine ${ }^{\circledR}$ and Uni-GoldTM), with a third (Hema StripTM or SD-Bioline) for discordant results. PCR was unavailable for definitive diagnosis in children $<18$ months of age, and CD4\% available only exceptionally.

HIV seropositive patients were referred for antiretroviral (ARV) medication based on clinical staging criteria, or occasionally on CD4\%. A waiting list for ARVs at the time of the study meant that these were rarely started during therapeutic feeding. Very few children were known to be HIV infected prior to admission.

Clinical and demographic information was collected on the day of admission or the next day after admission using a structured questionnaire administered by trained study staff. Data was entered using Epidata 3.1 (EpiData Association, Odense, Denmark, 2003-4). WHO Anthro 2005 v1 (World Health Organization, Geneva) was used to calculate anthropometric Z-scores using the NCHS reference. Odds ratios and independent t-tests were performed using SPSSv15 (C) SPSS Inc., USA) and StatCalc (CDC, Atlanta, USA, 1993).

The study was approved by the College of Medicine Research Ethics Committee.

\section{Results}

All 1024 children admitted to the NRU between July 2006 and March 2007 were included in the study. An additional 163 readmissions were recorded, but for this analysis, only first episode was considered.

All were offered HCT for the child, and mother (when present). An HIV result was available for $88.3 \%$ of admissions, with $459(45 \%)$ children seronegative and 445 $(43 \%)$ seropositive. The HIV status of 120 children $(11.7 \%)$ was not known, usually as the child had died early during admission. The mean age of admission was 30 months in the $\mathrm{HIV}$ infected, one month older than in the uninfected. There was a wider distribution of ages in the HIV infected children, with greater proportions of both infants, and children over 5 years.

\section{Clinical features on examination}

Clinical signs and symptoms according to HIV status are shown in table 1, presented by strength of association.

HIV was most strongly associated with chronic suppurative otitis media (CSOM) with an odds ration (OR) of 14.6 (95\% CI 5.8-36.7). Lymphadenopathy (OR 6.4, 95\%CI 3.511.7), clubbing (4.93, 2.63-9.4), marasmus (4.8, 3.53-6.83), hepato-splenomegally $(3.2,1.8-5.6)$, and oral candida (2.4, 1.8-3.27) had the next strongest associations. Any one of these signs was present in $74 \%$ of the HIV seropositive, though $38 \%$ of HIV uninfected children also presented with one of these. More than half the children with HIV presented with kwashiorkor.

Malnutrition was more severe in HIV infected children (tables 2 and 3), who were more underweight for age (mean $\mathrm{Z}$ score difference $[\Delta \mathrm{Z}] 0.49)$, more wasted $(\Delta \mathrm{Z} 0.73)$, and had more severe stunting $(\Delta Z$ 0.84). Significantly more were underweight, with $79 \%$ below the third percentile weight for age, compared to $53 \%$ of uninfected children.

Only children infected with HIV had Kaposis sarcoma (4), or Herpes Zoster (1), and 13 of the 15 children with parotid swelling were HIV seropositive.

\section{History of previous health problems}

Evidence of chronic illness was determined by number of hospital admissions, or OPD attendances in the previous 6 months. Both were significantly more common in the HIV infected, with $29 \%$ reporting a previous hospital admission, compared to $12 \%$. In the 3 months prior to admission $49 \%$ reported more than 3 OPD attendances compared to $25 \%$ in the HIV seronegative. Recurrent respiratory infections were reported in $18 \%$ of those with $\mathrm{HIV}$, compared to $2 \%$, and persistent fever was twice as common $(12 \%)$ as in the HIV uninfected. Persistent diarrhoea was not associated with HIV $(p=0.46)$.

\section{Family history}

Maternal orphaning was reported in 11\% of HIV seropositive children, and loss of a father in $9.9 \%$, double the rates in the HIV uninfected. A household contact with TB was more common $(14.4 \%$ v.s. $8.9 \%)$, as was a past history TB treatment in the child.

\section{Laboratory investigations}

There was only limited laboratory analysis available for comparison, as only packed cell volume (PCV) and malaria films were routinely available. PCV was $30.4 \%$ in HIV, significantly lower than in the uninfected $(32 \%)$. Malaria parasitaemia was less common in HIV, though not significantly so $(p=0.09)$.

\section{Discussion}

This is one of the largest studies describing clinical features of underlying HIV infection in children presenting with SAM. It is important to note its limitations. Firstly in children under 18 months of age PCR confirmation of infection was not available. Serology may reflect maternal antibody, although by 15 months this is usually undetectable in the HIV uninfected. This would have caused some uninfected (HIV exposed) children to be misclassified. We chose to include these children, as clinical experience is that most are serologically confirmed as HIV infected after 18 months, or on PCR. To exclude them would have missed an important group of children presenting with SAM, and where PCR or CD4 testing are not available. WHO recommends use of clinical features to make a presumptive diagnosis of HIV (14), though malnutrition is not always a particularly sensitive marker of HIV in infants(31).

Secondly the level of immune impairment in the HIV infected was not assessed in this study. Recent data from two NRU studies $(17,19)$ suggests $69-85 \%$ of children with SAM and 
HIV have a CD4\% indicating severe immunodeficiency(14).

HCT was routinely offered in the NRU, and was found acceptable by families, with uptake by almost $90 \%$ of carers, a level similar to recent reports elsewhere in Malawi (3, 28, 32). This high uptake makes it unlikely that there would be significant selection bias in the study.

Are our findings generalisable to other settings? These results are from a region where HIV occurs commonly in SAM, and where food insecurity is endemic and often localized, causing marked geographical and seasonal variation in the presentation and prevalence of HIV in malnutrition programmes(3). The pattern of SAM in Malawi is predominantly as kwashiorkor, which is less prevalent outside sub Saharan Africa. In addition the NRU was attached to an urban tertiary hospital, though over $95 \%$ of admissions came from the local district. There appear to be differences in the presentation of SAM in different NRUs, with some studies reporting much higher prevalence of diarrhoea $(13,26)$, possibly due to poorer water supply than available in Blantyre. These may influence the presentation of HIV in SAM, though our results are broadly in keeping with previously published studies.

\section{Clinical signs predictive of HIV}

We found CSOM to have the strongest association with HIV. This has been reported as more common in SAM in Zimbabwe (24), and noted in to be associated with HIV (2) on the paediatric wards in Malawi. Recurrent respiratory infections are a staging criteria in $\mathrm{HIV}(14)$, so it might be expected that previous ill health and more frequent medical attendance, would also be associated with HIV.

Lymphadenopathy was the sign most strongly associated with HIV in SAM in Burkino Faso (13) with sensitivity of $25 \%$ and specificity of $96 \%$, a similar finding to that in Zimbabwe (24). Clubbing indicative of chronic lung disease, has been reported as and predictive of HIV (2), though not reported in SAM. Prazuck reported a strong association with hepatomegally(13), though Kessler(18) in Malawi found no difference in prevalence of this. Although we identified a significant association with oral candida, this occurred in over $20 \%$ of uninfected children, making it an unreliable marker of HIV infection in SAM. Candida is commonly cited as associated with $\operatorname{HIV}(2,13,22-24)$.

Many studies have found persistent diarrhoea to be much more common in paediatric $\operatorname{HIV}(2,25,33)$ with a relative risk of 6.1 reported on the wards in Blantyre (2). However we found similar levels in children with SAM, present in $21 \%$ in HIV uninfected children and $23 \%$ of those with HIV. Others have similarly reported no differences in diarrhoea prevalence in $\operatorname{SAM}(13,18,21,24)$.

\section{Anthropometry}

Although marasmus is strongly associated with HIV, previous reports had implied that oedematous malnutrition was uncommon in HIV. The majority (54\%) of children with HIV in this study presented with oedema, consistent with reports of oedema in $67 \%$ and $71 \%$ from Malawi $(6,18)$ and $50 \%$ in Zambia(17), though rates were lower in Burkina Faso and Uganda at $37-43 \%(13,21,34)$.

Children with HIV were significantly more wasted, stunted and underweight, with twice the proportion of uninfected children being $<-4$ standard deviation $(\mathrm{Z})$ scores for each of these parameters. This reflects the chronic nature of HIV, with larger nutritional deficits in the HIV infected. Modification of the standard treatment guidelines for SAM may be appropriate, and knowledge of HIV status is therefore important(5). Children recover less quickly with HIV (35), and weight gain is associated with degree of immunodeficiency (19).

\section{Previous Clinical and Family History}

The clinical and family histories have not been addressed in previous studies, but are important tools in assessing patients. We found a history of recent admissions, and more than 3 outpatient attendances in the previous 6 months, to be predictive of HIV. Also associated were a history of persistent fever, recurrent respiratory infections, previous TB treatment, or a household contact with TB. Non-governmental and civil society organisations.

\section{Breastfeeding}

For children under 2 years of age, there was a 3 fold greater risk of being HIV seropositive if still breastfed. Although this could reflect reduced maternal to child transmission of HIV with early cessation of breastfeeding(36), most mothers were unaware of their HIV status. A more likely explanation is that HIV uninfected children rarely present whilst breastfeeding, as breast milk is an excellent source of nutrition, and protects against diarrhoeal and respiratory infections. Weaning the child off the breast is commonly associated with the development of SAM. Kessler(18) in Blantyre found a similar association with breastfeeding in HIV, although did not disaggregated for age under 2 years. In Zimbabwean children under 15 months no significant association with breastfeeding was found(24).

\section{Orphaning}

Orphaning has been reported to be associated with poorer outcomes in children born to mothers with HIV, irrespective of their HIV status(8). This has only been reported in one study on malnutrition, and was no more frequent in HIV (24), possibly as orphaning has a large impact on survival of all children, as it impacts on food security and care. We found children with HIV twice as likely to have lost either mother or father, however this had also occurred in $8.8 \%$ of the HIV uninfected.

\section{Laboratory}

Anaemia was more severe, with a small but significantly lower PCV observed in the HIV infected. This corresponds to a difference of $0.5 \mathrm{~g} / \mathrm{dl}$ haemoglobin, similar to the $0.6 \mathrm{~g} / \mathrm{dl}$ reduction previously noted in Malawi (28), but greater than that in Uganda(21). This does not appear to be due to malaria, which occurred less frequently in the HIV infected.

\section{Conclusion}

We have identified those clinical features strongly associated with HIV in severe malnutrition. These signs may allow some children with HIV to be better recognized when admitted to the ward. However in this study many of these features occurred in SAM not related to HIV.

The common distinguishing features in HIV infected children without malnutrition include persistent diarrhoea 
and oral candidiasis, both of which are frequently observed in SAM. In severe malnutrition CSOM, lymphadenopathy, clubbing, and hepatosplenomegally were found to be of greatest predictive of HIV, as was the presence of severe stunting or wasting. A child still breastfeeding, orphaned, or with a history of recent admission or clinic attendances, is also suggestive of HIV infection, as is a history of TB in the family. Most of these features had Odds Ratios $>2$ and a prevalence in the HIV infected child over 10\%, making them sufficiently common to be of use in assessing patients for features of HIV.

These features had significant overlap in SAM and HIV, and many severely malnourished children with HIV have none of these features present. In view of this we would recommend offering HCT to all children admitted with SAM in HIV prevalent environments. In our experience, this approach was acceptable to families. The ability to link families to HIV services is critical to high HCT uptake, as it can be explained to the family that there are specific, life prolonging treatments available, making knowledge of HIV status beneficial to both child and family.

\section{Acknowledgements}

We are grateful for the support received from the staff on Moyo House NRU at Queen Elizabeth Central Hospital, Blantyre, in particular to Sisters H. Khengere, A. Malamulo and C. Mhango, who helped supervise completion of patient admission forms; to Professors T Heikens and E Molyneux, for sharing their wisdom on how to care of children with SAM and HIV; and to S Collins and P Bahwere for allowing this work to be nested with the Pronut study. We are particularly thankful to H. Blencowe for assistance with data entry, and unwavering support to the project team.

\section{References}

1. UNICEF. The State of African Children 2008. 2008: 1-54.

2. Rogerson SR, Gladstone M, Callaghan M, et al. HIV infection among paediatric in-patients in Blantyre, Malawi. Trans R Soc Trop Med Hyg. 2004; 98:544-52.

3. Thurstans S, Kerac M, Maleta K, Banda T and Nesbitt A. HIV prevalence in severely malnourished children admitted to nutrition rehabilitation units in Malawi: geographical \& seasonal variations a cross-sectional study. BMC Pediatr. 2008; 8:22.

4. Fergusson $\mathrm{P}$ and Tomkins A. HIV prevalence and mortality among children undergoing treatment for severe acute malnutrition in subSaharan Africa: a systematic review and meta-analysis. Trans R Soc Trop Med Hyg. 2009; 103:541-8.

5. Heikens GT, Bunn J, Amadi B, et al. Case management of HIVinfected severely malnourished children: challenges in the area of highest prevalence. Lancet. 2008; 371:1305-7.

6. Chintu C, Bhat GJ, Walker AS, et al. Co-trimoxazole as prophylaxis against opportunistic infections in HIV-infected Zambian children (CHAP): a double-blind randomised placebo-controlled trial. Lancet. 2004; 364:1865-71.

7. Sutcliffe CG, van Dijk JH, Bolton C, Persaud D and Moss WJ. Effectiveness of antiretroviral therapy among HIV-infected children in sub-Saharan Africa. Lancet Infect Dis. 2008; 8:477-89.

8. Mermin J, Were W, Ekwaru JP, et al. Mortality in HIV-infected Ugandan adults receiving antiretroviral treatment and survival of their
HIV-uninfected children: a prospective cohort study. Lancet. 2008; 371:752-9.

9. Beisel WR. Nutrition in pediatric HIV infection: setting the research agenda. Nutrition and immune function: overview. J Nutr. 1996; 126:2611S-5S

10. Chandra RK. Numerical and functional deficiency in T helper cells in protein energy malnutrition. Clin Exp Immunol. 1983; 51:126-32.

11. Arapadi S. Growth failure in HIV infected children. WHO, Geneva; 2005.

12. Waterlow JC, Tomkins A and Grantham-McGregor SM. Proteinenergy malnutrition. London: Edward Arnold; 1992.

13. Prazuck T, Tall F, Nacro B, et al. HIV infection and severe malnutrition: a clinical and epidemiological study in Burkina Faso. AIDS. 1993; 7:103-8.

14. WHO. Antiretroviral therapy of HIV infection in infants and children: towards universal access Recommendations for a public health approach. Geneva: WHO; 2007.

15. Bachou H, Tumwine JK, Mwadime RKN and Tylleskar T. Risk factors in hospital deaths in severely malnourished children in Kampala, Uganda. BMC Pediatrics. 2006; 6:7.

16. Yeung S, Wilkinson D, Escott S and Gilks CF. Paediatric HIV infection in a rural South African district hospital. J Trop Pediatr. 2000; 46:107-10.

17. Hughes SM, Amadi B, Mwiya M, et al. CD4 counts decline despite nutritional recovery in HIV-infected Zambian children with severe malnutrition. Pediatrics. 2009; 123:e347-51.

18. Kessler L, Daley H, Malenga G and Graham S. The impact of the human immunodeficiency virus type 1 on the management of severe malnutrition in Malawi. Ann Trop Paediatr. 2000; 20:50-6.

19. Fergusson P, Chinkhumba J, Grijalva-Eternod C, Banda T, Mkangama $\mathrm{C}$ and Tomkins A. Nutritional recovery in HIV-infected and HIV-uninfected children with severe acute malnutrition. Arch Dis Child. 2009; 94:512-6.

20. Sadler K, Kerac M, Collins S, Khengere H and Nesbitt A. Improving the management of severe acute malnutrition in an area of high HIV prevalence. J Trop Pediatr. 2008; 54:364-9.

21. Bachou H, Tylleskar T, Downing R and Tumwine JK. Severe malnutrition with and without HIV-1 infection in hospitalised children in Kampala, Uganda: differences in clinical features, haematological findings and CD4+ cell counts. Nutr J. 2006; 5:27.

22. Karande S, Bhalke S, Kelkar A, Ahuja S, Kulkarni M and Mathur M. Utility of clinically-directed selective screening to diagnose HIV infection in hospitalized children in Bombay, India. J Trop Pediatr. 2002; 48:149-55.

23. Sunguya BF, Koola JI and Atkinson S. Infections associated with severe malnutrition among hospitalised children in East Africa. Tanzania Health Research Bulletin. 2006; 8:189-92.

24. Ticklay IM, Nathoo KJ, Siziya S and Brady JP. HIV infection in malnourished children in Harare, Zimbabwe. East Afr Med J. 1997; $74: 217-20$.

25. Chintu C, Dupont HL, Kaile T, et al. Human immunodeficiency virus-associated diarrhea and wasting in Zambia: selected risk factors and clinical associations. Am J Trop Med Hyg. 1998; 59:38-41.

26. Amadi B, Kelly P, Mwiya M, et al. Intestinal and systemic infection, HIV, and mortality in Zambian children with persistent diarrhea and malnutrition. J Pediatr Gastroenterol Nutr. 2001; 32:550-4.

27.Hosseinipour MC, Napravnik S, Joaki G, et al. HIV and parasitic infection and the effect of treatment among adult outpatients in Malawi. J Infect Dis. 2007; 195:1278-82.

28. Chinkhumba J, Tomkins A, Banda T, Mkangama C and Fergus- 
son P. The impact of HIV on mortality during in-patient rehabilitation of severely malnourished children in Malawi. Trans R Soc Trop Med Hyg. 2008; 102:639-44.

29. Kerac M, Bunn J, Seal A, et al. Probiotics and prebiotics for severe acute malnutrition (PRONUT study): a double-blind efficacy randomised controlled trial in Malawi. Lancet. 2009; 374:136-44.

30. MoH Malawi. Interim Guidelines for the management of acute malnutrition through Community-based Therapeutic Care. 2007.

31. Peltier CA, Omes C, Ndimubanzi PC, et al. Validation of 2006 WHO prediction scores for true HIV infection in children less than 18 months with a positive serological HIV test. PLoS ONE. 2009; 4:e5312.

32. Bahwere P, Piwoz E, Joshua MC, et al. Uptake of HIV testing and outcomes within a Community-based Therapeutic Care (CTC) programme to treat severe acute malnutrition in Malawi: a descriptive study. BMC Infect Dis. 2008; 8:106.

33. Cegielski JP, Msengi AE, Dukes CS, et al. Intestinal parasites and HIV infection in Tanzanian children with chronic diarrhea. Aids. 1993; 7:213-21.

34. Babirekere-Iriso E, Musoke P and Kekitiinwa A. Bacteraemia in severely malnourished children in an HIV-endemic setting. Ann Trop Paediatr. 2006; 26:319-28.

35. Ndekha MJ, Manary MJ, Ashorn P and Briend A. Home-based therapy with ready-to-use therapeutic food is of benefit to malnourished, HIV-infected Malawian children. Acta Paediatr. 2005; 94:222-5.

36. Coutsoudis A, Dabis F, Fawzi W, et al. Late postnatal transmission of HIV-1 in breast-fed children: an individual patient data meta-analysis. J Infect Dis. 2004; 189:2154-66. 